\title{
An Agent-based Architecture for Advance Reservations
}

\author{
Olov Schelén and Stephen Pink \\ Computer Science and Electrical Engineering \\ Luleå University of Technology \\ S - 97187 Luleå, Sweden \\ \{olov, steve\}@cdt.luth.se \\ Tel: +4692091000 Fax: +4692072821
}

Keywords: Advance Reservations, Reservation Agents, Quality of Service

\begin{abstract}
We propose an architecture where clients can make advance reservations through agents. For each routing domain in the network there will be an agent responsible for admission control on behalf of the routers in the domain. Requests involving several routing domains are forwarded for admission control with agents along the path for the requested service. Agents maintain hard reservation state using a reliable protocol for agent intercommunication.

Agents start allocating resources for advance reservations in the routers by setting up forwarding state shortly before resources are needed for packet forwarding. Resources are made available for advance reservations by means of rejecting further immediate requests and ultimately by preempting some immediate reservations. We have shown that the risk of preemption can be kept very low.

Thus, agents can set up packet classifiers and schedulers in their routers, allowing routers to get on with their main task, packet forwarding.
\end{abstract}

\section{Introduction}

Real-time applications such as audio and video conferencing may need resource reservations in the network to perform well. However, many current resource reservation proposals allow only immediate reservations, i.e., reservations at the time the session begins, while real-time events often are scheduled and advertized in advance. Support for advance reservations allows both the network and its users to more

\footnotetext{
${ }^{0}$ This work was supported by a grant from the Center for Distance Spanning Technology (CDT), Luleå, Sweden
}

effectively schedule resources when the time of the future event needing reservations is known in advance.

Current protocols designed for resource reservations such as RSVP [29] and the ATM signaling protocol Q.2931 [31], and its Internet analog ST-2 [25] are designed for immediate reservations. In [10], we explain how to extend the soft-state approach in RSVP for support of advance reservations. However, using these protocols for advance reservations would be difficult since senders and receivers would have to be in place and capable of making reservations and large amounts of state would have to be stored in the network for potentially long periods of time.

In this paper we present an architecture where advance reservations are provided by reservation agents. For each routing domain in the network, there will be a reservation agent responsible for admission control on behalf of the routers in the domain (figure 1). Users can make reservation requests by contacting any reservation agent. Reservation requests are forwarded to the agent responsible for the routing domain concerned by the request.

Requests involving several routing domains are forwarded for admission control to the agents along the path. Agents can avoid forwarding each request along the path by making long-term bulk reservations with other agents so that admission requests over several routing domains can be granted locally. Agents establish reservation state through reliable handshaking.

When the time to make resources available approaches, reservation agents reserve resources in routers by setting up forwarding state along the path. A routers primary task is packet forwarding, i.e., packet classification and scheduling. Agents are responsible for setting up appropriate forwarding state in the 


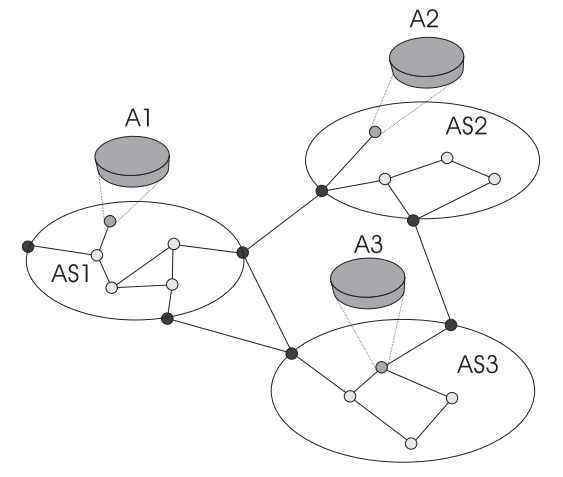

Figure 1: Routing domains (AS1 - AS3) and reservation agents (A1 - A3)

routers when it is needed.

An advantage with an agent-based approach is that advance reservations can be provided with little support from routers and without having to maintain reservation state in routers through periods when reservations have no effect on packet forwarding. Furthermore, the agent-driven approach does not require reserving endpoints to be present until the session for which resources have been reserved is starting. Reservations can also be made for remote locations, thereby supporting nomadic computing, i.e., reservations for places where the host will be moving in the future. Since reservations are maintained by agents, reservation requests can be made by a third party, who may make reservations for several endpoints. Thus, neither senders nor receivers need to be in place when resource reservations are made.

\section{Overview of the architecture}

The Internet has relied on a more-or-less stateless architecture for providing best-effort service. Softstate solutions such as RSVP have been proposed to provide immediate reservations in an otherwise stateless network. However, advance reservations do not fit into this model because of the amount of overhead that can be generated over long periods of time.

The basic principle of soft-state is that state is refreshed periodically by endpoints either by sending explicit state setup messages or implicitly by sending data packets. This approach works for state that is deployed only during periods when it is actually used for packet forwarding. Many policies such as immediate resource reservations have this property. The signaling overhead for maintaining state is marginal, especially if policies don't change very frequently. The refresh frequency can be low as route changes will be detected locally and trigger immediate forwarding of messages for setting up state over new paths. For immediate reservations this scheme is efficient and flexible.

However, since we expect the book-ahead time for advance reservations to be longer than the actual duration of the session, the relative signaling overhead for maintaining advance reservation state may become unacceptable. We propose that agents, deployed in end systems, maintain state about reservations that will be needed for future packet forwarding and avoid deploying state in routers until there are packets in the network whose forwarding depends on the reservation.

A reservation agent is a host that is responsible for reserving resources on a part of the Internet. Typically there is one reservation agent responsible for each autonomous system (AS) in the Internet (an AS is an administrative domain containing a number of routers running a routing protocol internally). Such reservation agents advertize themselves periodically on a well known multicast address, giving their identity and which routing domain (AS) they are responsible for. By listening to this well known multicast address, each top level agent will know the existence of all other top level agents. Inside an AS there may be backup agents as well as a hierarchy of agents, e.g., different agents for different areas of the AS. Most agents are internal, i.e., not visible outside the AS, but if there is an agent failure, one of the backup agents is advertized instead.

Each reservation agent must know the topology of its routing domain to perform admission control on behalf of that domain, and also to detect topology changes that require re-computation of forwarding state setup in routers, or renegotiation with adjacent reservation agents. This topology information can be obtained through a link state routing protocol that provides each node, including the agent, with a topology database. It is in the nature of hierarchical routing that an agent cannot have knowledge about the internals of remote autonomous systems and therefore can only provide admission control for it's own routing domain. Thus, we need at least one agent for each routing domain.

For agent-based resource reservations, static link performance measures such as link capacity must be known. In the present version of OSPF [20], there are link metrics for different type of services to allow for type-of-service (tos) routing, but there is no information on link capacity. In QOSPF [30], both static link resources and dynamic available resources are advertized. In our scheme, only static link performance is 
necessary. This information could be included in link state advertisements, which would require an addition to the OSPF specification. Instead we suggest using SNMP for finding out specific properties of links. This solution does not require any changes to the link-state routing protocol, and allows agents to monitor any information made available through SNMP.

To avoid undue signaling overhead and scalability problems, agents do not measure traffic dynamics. Admission control in agents is parameter-based, i.e., considering static link resources and traffic descriptions given in admission requests. Agents may setup resources along shortest paths or perform QoS routing based on the parameters in the admission requests. QoS routes are installed in the forwarding state of the routers.

The architectural goal is to relieve the routers from admission control and other QoS related computing as far as possible. Routers must have an interface so that agents can set up packet forwarding directly. This can be accomplished through SNMP and a special management information base.

For reservation agents to find out which autonomous systems are on the path between source and destination, agents need access to routing information that resides in the border routers of their autonomous system. This can be accomplished either by getting routing information from a border router through SNMP, or by having border routers inject external routing information into their AS. The former may be suitable for stub ASes where no external routing information is injected, while the latter may be suitable for ASes with more than one border router. External routing information together with agent advertisements can be used for forwarding admission requests to other agents.

\subsection{Mixing advance and immediate ad- mission control}

Agents also support immediate reservations. Network resources are available for both advance and immediate reservations. Advance requests specify a duration of the requested service while immediate requests do not specify durations. In case of conflicts due to unknown durations, immediate requests may be preempted.

In a related paper [24], we present simulation results on sharing network resources between advance and immediate admission control when the admission control algorithms are loosely coupled. Advance admission control is performed without considering current immediate reservations. Immediate admission control can be performed without considering advance reservations, but the accuracy will be increased by considering resources to be allocated for advance reservations in the near future. Resources are made available for advance reservations by blocking immediate resource requests, or ultimately by means of preempting service for flows that made immediate reservations. In [24], we present results on resource utilization, rejection probability and preemption probability when resources are shared according to this model.

The results show that admission control agents can handle advance and immediate parameter-based admission control, or alternatively coexist with a separate mechanism for immediate admission control, e.g., the IETF int-serv model where immediate admission control (guaranteed service or controlled load service) is performed in routers.

\section{Advance reservations over several domains}

Agents can interoperate to support end-to-end advance reservation requests in the global network. A host requesting a reservation may consult any reservation agent, e.g., the local one or the favorite agent, just as one may use different travel agents for booking airline tickets. Some agents may already have records for managing accounting etc.

\subsection{Unicast}

A typical unicast advance request includes a duration, a traffic specification, a source, and a destination. There is no notion of sender or receiver associated with the node making the request. The source and destination descriptions may be the addresses of a sending host and a receiving host respectively. However, in many cases we don't know the addresses of endpoints at the time of making the reservation, but we may know the local area network where addresses temporarily acquired during a session. This may be the case for mobile or stationary computers that acquire addresses through a dynamic host configuration protocol (DHCP), or for computers that will be setup for a specific event, e.g., for an IETF conference. An address mask associated with each endpoint address will solve this problem. Address masks also provide a mechanism for making aggregate reservations, i.e., bulk reservations between different domains of the network.

\subsection{Multicast}

A multicast advance reservation consists of a duration, a multicast address and a list of senders and re- 
ceivers respectively. Each sender and receiver is specified by an address and an address mask. A multicast reservation tree is identified by its multicast destination address and can be extended by making further reservations for the same multicast address. Overlapping requests, i.e., requests having links in common, is merged by the reservation agent forming one shared multicast tree.

\subsubsection{Rendezvous agents}

To support advance reservations with little overhead we have a rendezvous agent associated with each multicast address. The rendezvous agent has group membership information, i.e., the complete list of senders and receivers. When making advance multicast reservations, the rendezvous agent provides an updated list of participants so that multicast reservations can be made, starting from the agents responsible for receiver domains. Building the tree starting at receivers is more efficient as sessions often involve fewer senders than receivers, and adding a receiver may result in reservations over a few additional links for joining an already established multicast reservation tree.

Some current multicast routing protocols, e.g., sparse mode Protocol Independent Multicast [8] and Core Based Trees [1], use rendezvous points. They are designed for setting up multicast routing trees immediately, i.e., when receivers are in place and communicating. In PIM a rendezvous point only keeps track of senders of the group and each receiver (local router) is responsible for joining with the rendezvous point to obtain a branch of the multicast tree. PIM also allows the multicast tree to be rebuilt on demand from individual receivers (local routers) to use shortest paths. To support new receivers joining, the rendezvous point is always a member of the multicast tree (thus receiving data from all senders).

Our notion of rendezvous agents is different. First to support reservations for not yet present receivers, a rendezvous agent must record all members of the group. i.e., both senders and receivers. Second, the rendezvous agent is only used as a database for finding group membership. There is no obligation that sender data pass the rendezvous agent. Group membership information is used to build multicast trees that will finally be used so that resource reservations can be made on the right branches. It does not make sense to change the distribution tree on demand when there are advance reservations, unless there are sudden changes in topology making it impossible to meet committed service along the chosen paths. The decision about whether to build a shortest-path tree or a core-based tree can be taken by the agents. We leave this issue open for now.

\subsubsection{Hierarchical agents}

To support scaling, we use a hierarchical approach, i.e., agents register border points of their routing domains rather than specific endpoints. The internal multicast tree and the local members of a routing domain are concealed by the reservation agent. Adding another member to a session where members are sharing resources can be done internally. Inside an AS there is a hierarchy of routing domains and there will be a corresponding hierarchy of reservation agents that aggregate reservations and register with the next highest level. At the top level there are several ASes and corresponding peer agents. These agents should only register involved border routers of their autonomous systems with the rendezvous agent. The task of global rendezvous agents is to maintain the list of member ASes of their multicast groups. Hierarchical approaches have been suggested before, in [15] a hierarchical approach for organizing PIM rendezvous points is suggested.

The scalability problem could be approached by getting rid of rendezvous agents completely. In densemode schemes for building immediate multicast trees a flood and prune approach is often used [9] [26]. However, for advance reservations there is no data available until the session starts and therefore the relative cost of flooding control information between agents and keeping prune state during the booking time would be high.

A related issue is how to find the rendezvous agent for a given multicast address. With a hierarchical approach, all agents know about all other agents at the same level (by multicasting), so the problem boils down to partitioning the address space between agents. We propose an approach based on dynamic prefix partitioning of multicast addresses. The range of multicast addresses can be advertised as an address prefix associated with each agent. Agents then need a distributed algorithm to partition a multicast address space between themselves. The solution requires that a range of consecutive multicast addresses is allocated for the agent architecture.

\section{Packet classification}

Deciding which packets that are to use against a particular reservation is known as packet classification. This is performed by the routers for each packet and therefore poses a scaling problem. There are basically two ways of doing packet classification. 
First, a filter specification could be given by clients having reserved resources. Packet classification is performed on the headers of the packets. In RSVP filtering is performed primarily on addresses and destination and source ports. Second, a reservation key (a certificate) could be included in each packet that is to use against a reservation. Packet classification is done on this single field of the header. Depending on how many different service classes we support, the field could be one or many bits long.

The problem with filter specifications is efficiency. In the worst case of filtering on sender addresses, the routers need a filter for each sender to a multicast group. Multicast routing does not scale well even without packet classification, i.e., for best effort service. Current proposals for multicast routing scales at best as $O(G)$, where $G$ is the number of multicast groups that are served by a router [1]. Thus, the size of the multicast routing table is proportional to the number of multicast addresses that are currently served by the router.

Our goal is to add multicast resource reservations while preserving the $O(G)$ scaling factor also for packet classification. This rules out making classification on specific source addresses. Instead we suggest that the rendezvous agent issue a key for the multicast address to other trusted agents, which in their turn may install the key in routers. The key may be added to packets by clients having reservations for sending to that group, or for better security, by the most local router under authority of a reservation agent. In the latter case only the local router must do classification and policing on packet headers to decide which packets that should be annotated with reservation keys. In IPv6, keys could be included in the hop-by-hop options header. Since agents can compute one aggregate reservation for each hop of the multicast tree, routers can make classification using only one key for each reservation.

This scheme provides scalable support for aggregate multicast reservations, i.e., where a set of senders share reserved resources. The amount of state needed ed for packet classification in routers is in $O(G)$, which is comparable with best-effort multicast routing. The RSVP model supports a stronger but unscalable model where it is possible to reserve resources in routers for individual senders (known as fixed filters) or for a set of senders (known as shared explicit filter). One motive for having this support is for receivers with insufficient network resources to be able to allocate resources only for the senders that it is interested in. However, in a typical multicast session the set of interesting senders changes dynamically, e.g., with the current speaker of a meeting, so a receiver with insufficient network resources would have to make a lot of re-reservations during the session. Another motive is for misbehaving senders not to be able to steal bandwidth from reservations, i.e., some policing is done by packet classification.

In our model, applications must make sure to use multicast addresses so that independently of the number of sources there is a limit on the bandwidth that is used by the aggregate multicast flow. Audio and video servers, usually have a known bandwidth limit, e.g., they provide flows with different bandwidths that can be selected by receivers depending on the capacity of their access link. It is important to know which maximum bandwidth that is used by a flow before connecting. In multiparty audio sessions bandwidth is limited by senders taking turns speaking. Video applications can be designed in a similar way, e.g., so that only the most recent speakers and the upcoming speakers on the speakers list send motion video. We believe that it is a bad idea to introduce nonscalable network solutions in order to support applications that waste bandwidth.

The advantage of providing shared reservations only is that packet classification can be done using only one classification entry for each flow (multicast address). This together with the possibility for making bulk reservations between subdomains of the network by using address prefixes to specify leaves of the reservation tree should provide a scalable mechanism for quality of service.

\subsection{Classification granularity}

Agents may chose internally how many different service levels to support. The low end extreme is to have only one admission controlled service level shared between all flows. Then, packets only need to carry one bit to tell whether it is to use the primary service. Routers need only one queue for the primary service and another queue for best effort service. Several service levels can be supported by having a fixed number of queues in routers. The high end extreme would be to allow a queue for each flow, e.g., flow-wise weighted fair queuing. The scaling for this may be acceptable for multicast, as multicast routing already keeps perflow state. For unicast, however, we suggest using a small number of admission controlled service levels to avoid per-flow state in the routers. If QoS routing is to be performed we may add service classes to distinguish between outgoing interfaces. 


\section{$5 \quad$ Security and policing}

In our architecture, packet classification is performed by including a reservation key in each packet that is using against a reservation. Consequently, malicious senders may take currently used keys and steal bandwidth from resource reservations. However, the agents know which senders are entitled to use reserved resources and occasional policing can be done to catch violating senders. Receivers of a multicast group may even hint to their provider when policing of a group should be done. The multicast reservation scheme should not include policing for unwanted senders at every hop as default. Instead, agents should have a way to setup routers close to the edges with specific policing tasks. When there is a malicious sender identified, an agent should be able to tell the most local trusted router of the malicious sender to exclude the flow. Thus, per source filtering and policing is introduced at specific points close to the edges of the network when necessary. Per-source policing does not scale well and therefore local routers cannot be expected to have sufficient capacity for policing continuously for every local sender. In the general case, policing should be soft in the sense that only a few flows are checked at each time. The policing policy is driven by the reservation agents. An important issue is to develop policing and protection mechanisms that are fast and scalable enough to find and exclude violating flows accurately. The requirements depend on how common deliberate stealing of reserved resources will be.

\section{Setting up forwarding state in routers}

Resource set-up messages are distributed by agents to routers just before the resources are allocated. We use a soft state setup protocol where agents contact and refresh each involved router in their domain periodically. Such a protocol can be optimized by using multicast in cases where there are many routers that are to be updated with the same message. Since soft state is refreshed periodically, we do not need a reliable multicast protocol. Instead the refresh frequency can start high as new state is deployed and then gradually decrease as there is no change in state.

If the overhead for soft state turns out to be too high, a hard state protocol could be used instead. Agents could open a TCP connection (unicast) to each of their routers and setup state reliably. With this approach the routers must be able to clear up reservation state in case a reservation agent should go down and forget about the state.

To allow reservation agents to query and set router state, the routers must expose an interface. For an initial implementation the most straight forward approach is to use SNMP, i.e., having agents setting router state by sending unicast SNMP messages to each of them. The main problem is to make necessary router state available through the management information base (MIB). The authentication problem will be solved by SNMP mechanisms.

\section{Routing performed by agents}

Agents can choose to follow best effort routes and establish forwarding state for reserved resources along the current path. However, sometimes routes change (or flap) although the old route is still available and has sufficient resources for the committed service.

We suggest that agents be responsible for selecting routes and that these routes can be included in the forwarding state set in routers by agents. This also makes quality of service (QoS) routing possible. For advance reservations there is time for calculating QoS routes. When allocating resources in routers, the agents provide the address/interface of the next hop as well. Thus, routing information is included in the packet classification state, so that flows using resources reserved through agents can take a different route than the best effort packets to the same destination.

It is the job of the agent to re-route at link or router failure. If there is a link failure, routers next to the failed link will detect the problem first, e.g., through the OSPF HELLO messages. This will trigger a link state advertisement to other nodes in the AS, including the reservation agent. As the reservation agent finds that a link is broken, it will process the reservations on that link by recomputing routes, checking if there is capacity left for advance reservations through alternative routes. This will work if the capacity is available and flows haven't yet started. If capacity on alternative routes is insufficient, the agent can just wait and retry, or choose to inform users who have made advance reservations about possible problems. If there are reservations for currently active flows on the broken link and there is capacity in an alternative path, the agent will immediately allocate resources on the alternative path. The old reservation state in the routers can be torn down by the agent or simply left to time out by not being refreshed.

Thus, when there are failures, routes will change only if there is an alternative path that can provide resources, otherwise the traffic is served best effort. 
When routes change the agents take multi hop decisions over the involved reservations, so the scheme does not suffer from having an arbitrary outcome in terms of who gets what on which hops. When links between different administrative domains fail, several reservation agents need to react.

As rerouting doesn't happen instantaneously, the flow state may direct packets for a short time to an interfaces that is unavailable. When this happens the packets should be sent through best effort routing instead. That includes removing any reservation keys in the packet so that best-effort service is used all the way to the destination. This works fine for unicast, but for multicast there may not be any best effort multicast routing state available and then the packets will be dropped.

If all routers along the path support the agent architecture, all routes are loop-free. However, routing loops may occur when there are routers on the path that only support best-effort routing. To avoid this, the flow state routing must check so that packets arrive on the correct incoming interface. Should a packet arrive on an incoming interface different from the one negotiated during QoS route setup, it may be a packet that coming into the QoS path again after a loop.

\section{Comparison with the RSVP policy architecture}

The RSVP policy architecture of [16] [17] is an extension to the RSVP architecture [29]. Reservation requests goes through both resource based admission control at routers and through policy based admission control managed by local policy modules or by policy servers. Policies are bilateral agreements between neighbor providers and policy admission control is enforced at border nodes only. Reservation requests, carried via the normal RSVP soft state mechanisms, contain policy data objects that are passed to local policy modules at border nodes. Policy data objects may be added, removed, or rewritten by policy modules along the path. Routers have the choice to handle policies in their local policy module or to outsource policy decisions to a policy server. There is a protocol for communication between routers and policy servers using hard or soft state.

An important difference between our approach and the RSVP policy approach is the role of routers. In our architecture, agents handshake to make reservations and to compute the forwarding state for routers, while the routers primary task is to do packet forwarding, i.e., best effort service and service disciplines installed by the agents. Admission control and QoS routing decisions are done by agents. Our goal has been to relieve the routers from computation concerned with QoS contracts and put it in agents instead. Thus admission control and service commitments are all managed by agents. In RSVP, routers play a central role in admission control and routing. Routing decisions are not part of RSVP but instead made by the best effort routing mechanisms in the routers. Consequently the RSVP mechanism can easily reestablish reservations along changing routes. However, when routes change (or flap) the outcome in terms of who gets reservations depend on the order reservations arrive on the new path. An annoying problem with RSVP is that users do not know if a reservation is granted end-toend. Non-RSVP routers may be on the path and may disturb end-to-end quality. We believe that RSVP is suitable for providing loose service commitments but it cannot support guaranteed service very well. Our architecture may adapt more slowly to changing topology but it provides a better vehicle for guaranteed quality of service and certainly for advance reservations (which it was designed for from the very beginning). In RSVP senders must be present to make reservations, which might not always be possible far in advance.

Another problem with RSVP is poor support for aggregate reservations. Endpoints make reservations on a per-flow basis and although reservation messages are merged up the multicast tree, we still have reservations per flow. In addition, packet filtering is designed so there may be classification state for each sender. This is clearly not scalable. In our agent architecture aggregation of reservations is included from the beginning. Classification is based on keys (certificates) and the leaves of the multicast reservation tree may be routing domains (address prefixes). There is work in RSVP on introducing address prefix aggregation (aka CIDR prefixes), but alone this is not enough to handle sparsely located senders to the same multicast group. Packet filtering/classification may still have to be done on a per sender basis.

\section{Conclusions}

Advance and immediate reservations can be supported by agents responsible for admission control on parts of the Internet. Our architecture minimizes disturbing the primary task of the routers: packet forwarding. Soft state in the routers is deployed only during periods when it is needed for packet forwarding.

The agent-driven approach described in this paper does not require the communicating endpoints to be 
present until the session for which resources have been reserved is starting. Reservations can also be made for remote locations, thereby supporting nomadic computing, i.e., reservations for places where the host will be moving in the future. Since reservations are maintained by agents, reservation requests can be made for a group of participants by a third party.

Reservation agents have access to routing databases for finding out the topology of the routing domain that it performs admission control for. Reservation agents use SNMP to find out the static link resources available in the network and for setting up forwarding state in the routers. Agents perform parameter-based admission control, using traffic descriptions provided by clients making reservation requests. Thus, agents work without signaling any traffic dynamics.

The agent architecture is suitable for parameterbased QoS routing of advance reservations. There is intrinsically more time available for admission control and QoS routing when requests are made in advance.

\section{References}

[1] A. Ballardie, P.F. Francis, J. Crowcroft. Core based trees. In Proceedings of the ACM SIGCOMM, San Francisco, 1993.

[2] A. Ballardie: Core Based Trees (CBT) Multicast Routing Architecture, Work in progress, May 1997.

[3] A. Banerjea, B. Mah: The Real-Time Channel Administration Protocol. Proc. 2nd International Workshop on Network and Operating System Support for Digital Audio and Video, November 1991.

[4] A. Banerjea, D. Ferrari, B. Mah, M. Moran, D. Verma, H. Zhang: The Tenet Real-Time Protocol Suite: Design, Implementation, and Experiences. Technical Report TR-94-059, ICSI, Berkeley, CA, November 1994.

[5] R. Braden, L. Zhang, S. Berson, S. Herzog, S. Jamin: Resource ReSerVation Protocol (RSVP) - Version 1 Functional Specification. Work in progress, Nov 1996.

[6] J. Case, K. McCloghrie, M. Rose, S. Waldbusser: Introduction to version 2 of the Internet-standard Network Management Framework. RFC 1441, April 1993.

[7] D. Clark, S. Shenker, L. Zhang: Supporting RealTime Applications in an Integrated Packet Services Network: Architecture and Mechanism. Proc. ACM SIGCOMM'92, 1992.

[8] S. Deering, D. Estrin, D. Farinacci, V. Jacobson, CG. Liu, L. Wei: An Architecture for Wide-Area Multicast Routing. Proc. ACM SIGCOMM'94, 1994.

[9] S. Deering, D. Estrin, D. Farinacci, V. Jacobson, A. Helmy, L. Wei: Protocol Independent Multicast Version 2, Dense Mode Specification, Work in progress, May, 1997.
[10] M. Degermark, T. Köhler, S. Pink, O. Schelén: Advance Reservations for Predictive Service. Proc. 5th NOSSDAV, Durham, New Hampshire, April 1995.

[11] D. Estrin, D. Farinacci, A. Helmy, D. Thaler, S. Deering, M. Handley, V. Jacobson, C. Liu, P. Sharma, L. Wei: Protocol Independent Multicast-Sparse Mode (PIM-SM): Protocol Specification, RFC 2117, June 1997.

[12] D. Ferrari: Real-Time Communication in an Internetwork. Journal of High Speed Networks, 1(1), 1992, pp. 79-103.

[13] D. Ferrari, A. Gupta, G. Ventre: Distributed advance reservation of real-time connections. Proc. 5th International Workshop on Network and Operating System Support for Digital Audio and Video, April 1995.

[14] A. Gupta: Advance reservations in real-time communication services. Sun Microsystems Laboratory. URL: http://www.cs.berkeley.edu/ amit/research/ Postscript/adv-issues.ps

[15] M. Handley, J. Crowcroft, I. Wakeman: Hierarchical Protocol Independent Multicast (HPIM), Oct 1995. URL ftp://cs.ucl.ac.uk/darpa/IDMR/hpim.ps

[16] S. Herzog:Building Blocks for Accounting and Access Control in RSVP: USC/ISI, November 1995.

[17] S. Herzog:RSVP Extensions for Policy Control. Work in progress, draft-ietf-rsvp-policy-ext-02.txt, April 1997.

[18] S. Jamin, P. Danzig, S. Shenker, L. Zhang: $A$ Measurement-based Admission Control Algorithm for Integrated Services Packet Networks. Proc. ACM SIGCOMM'95, 1995.

[19] D. J. Mitzel, D. Estrin, S. Shenker, L. Zhang: A Study of Reservation Dynamics in Integrated Services Packet Networks. Proc. IEEE INFOCOM'96, March 1996.

[20] J. Moy:OSPF Version 2. RFC 1583, March 1994.

[21] C. Partridge, S. Pink: An Implementation of the Revised Internet Stream Protocol. Internetworking Research and Experience, 3(1), March 1992, pp. 27-54.

[22] W. Reinhardt: Advance reservation of network resources for multimedia applications Proc. ICAWA 94, Germany, October 1994.

[23] Y. Rekhter, T. Li:A Border Gateway Protocol 4 (BGP-4) RFC 1654, July 1994.

[24] O. Schelén, S. Pink: Sharing Resources through Advance Reservation Agents. Proceedings from IWQoS'97, International Workshop on Quality of Service, New York, May 1997. URL: http://www.cdt. luth.se/ olov/publications.html

[25] C. Toplocic: Experimental Internet Stream Protocol, Version 2 (ST-II). RFC 1190, October 1990.

[26] D. Waitzman, C. Partridge, S. Deering: Distance Vector Multicast Routing Protocol. RFC 1075, November 1988 . 
[27] L. Wolf, L. Delgrossi, R. Steinmetz, S. Schaller, $\mathrm{H}$. Wittig: Issues of Reserving Resources in Advance. Proc. 5th International Workshop on Network and Operating System Support for Digital Audio and Video, April 1995.

[28] D. Zappala, B. Braden, D. Estrin, S. Shenker, D. Zappala: Interdomain Multicast Routing Support for Integrated Services Networks. November 1996.

[29] L. Zhang, S. Deering, D. Estrin, S. Shenker, D. Zappala: RSVP: A New Resource ReSerVation Protocol. IEEE Network Magazine, September 1993, pp. 8-18.
[30] Zhang, Sanches, Salkewicz, Crawley: Quality of Service Extensions to OSPF. work in progress, June 1996.

[31] "Broadband Integrated Service Digital Network (BISDN) Digital Subscriber Signaling System No.2 (DSS2) User Network Interface Layer 3 Specification for Basic Call/Connection Control", ITU-T Recommendation Q.2931, International Telecommunication Union: Geneva, 1994. 